\title{
Relações entre Bem-Estar Subjetivo e Satisfação Conjugal na Abordagem da Psicologia Positiva
}

\author{
Relations between Subjective Well-Being and Marital Satisfaction on the Approach \\ of Positive Psychology
}

\author{
Fabio Scorsolini-Comin ${ }^{a, b} \&$ Manoel Antônio dos Santos ${ }^{*, b}$ \\ ${ }^{a}$ Universidade Federal do Triângulo Mineiro, Uberaba, Brasil \\ $\&{ }^{b}$ Universidade de São Paulo, Ribeirão Preto, Brasil
}

\begin{abstract}
Resumo
Objetivou-se investigar as correlações existentes entre os três fatores do bem-estar subjetivo (BES) afetos positivos, afetos negativos e satisfação com a vida - e os três fatores da satisfação conjugal (SC) interação conjugal, aspectos estruturais e aspectos emocionais. Participaram 106 voluntários de ambos os sexos, com idade média de $42 \pm 11$ anos, casados e com tempo médio de união de 16,11 $\pm 11,35$ anos. Foram aplicadas as escalas de BES (EBES) e de Satisfação Conjugal (ESC). Os resultados foram analisados pela técnica de regressão múltipla stepwise. Não foram encontradas associações entre os fatores satisfação com a vida (fator do BES), aspectos emocionais (fator da SC), aspectos estruturais (SC) e interação conjugal (SC). O BES e a SC, fatores gerais, também não apresentaram associações significativas. Destaca-se o papel dos afetos positivos (fator do BES) na percepção da satisfação conjugal (SC), o que é congruente com o enfoque da Psicologia Positiva, que atesta que as pessoas que experimentam emoções positivas tendem a se engajar em relacionamentos mais satisfatórios.

Palavras-chave: Bem-Estar Subjetivo; Satisfação Conjugal; Escalas; Psicologia Positiva.

Abstract

The objective of the present study was to investigate the correlations between subjective well-being (SWB) and marital satisfaction (MS) among married people. A hundred six married people (53 couples) with an average age of $42 \mathrm{yrs}(S D=11)$ participated in the study. The following instruments were used: Subjective Well-being Scale and Marital Satisfaction Scale. In this study, correlation analysis and stepwise multiple regression analysis showed that there were no associations among satisfaction with life (SWB factor), emotional aspects (MS factor), structural aspects (MS factor) and marital interaction (MS factor). The SWB and the MS (general factors) were not significantly correlated. According to the findings of the current study, there is the role of positive affect (SWB factor) on the perception of MS, which is consistent with the approach of Positive Psychology. It attests that people who experience positive emotions tend to engage themselves in more satisfying relationships.

Keywords: Subjective Well-Being; Marital Satisfaction; Scales; Positive Psychology.
\end{abstract}

A família contemporânea tem passado por profundas transformações, o que exige que o olhar que dedicamos à intimidade da relação conjugal seja modificado, de modo a abranger os dinamismos familiares, compreendidos como os movimentos de mudanças e permanências, rupturas e continuidades ao longo do tempo (Jablonski, 2001; Mosmann, Wagner, \& Féres-Carneiro, 2006). Nesse sentido, tem-se concebido o casamen- to como um espaço no qual coexistem e atuam, simultaneamente, forças da conjugalidade e da individualidade. Embora no relacionamento conjugal os parceiros constituam uma área de compartilhamento de emoções, experiências, expectativas e desejos (conjugalidade), continuam a existir - e também a se modificar incessantemente -, as suas individualidades constitutivas (FéresCarneiro, 1998).
* Endereço para correspondência: Universidade de São Paulo, Faculdade de Filosofia, Ciências e Letras, Departamento de Psicologia, Avenida Bandeirantes, 3900, Monte Alegre, Ribeirão Preto, SP, Brasil, CEP 14040901. E-mail: scorsolini usp@yahoo.com.br Trabalho derivado da dissertação de mestrado intitulada "Casar, Verbo (In)Transitivo: Bem-estar Subjetivo, Conjugalidade e Satisfação Conjugal na Perspectiva da Psicologia Positiva", do primeiro autor, sob orientação do segundo autor, defendida e aprovada em 2009 junto ao
Programa de Pós-graduação em Psicologia da Faculdade de Filosofia, Ciências e Letras de Ribeirão Preto da Universidade de São Paulo. Os autores agradecem à Fundação de Amparo à Pesquisa do Estado de São Paulo (FAPESP) por ter subvencionado o projeto que originou o presente estudo, com bolsa de mestrado (Processo $n^{\circ}$ 2007/52584-5), e ao Conselho Nacional de Desenvolvimento Científico e Tecnológico (CNPq) pela bolsa de Produtividade em Pesquisa, nível 1D, concedida ao segundo autor. 
Nesse contexto, o objetivo deste estudo foi investigar as correlações existentes entre os fatores do bem-estar subjetivo (BES) e da satisfação conjugal (SC), compreendendo em que medida a avaliação do bem-estar está relacionada à vivência conjugal e vice-versa, a partir da utilização de escalas de avaliação de bem-estar individual (EBES) e satisfação conjugal (ESC), descritas ao longo do texto. Os conceitos que nortearam a investigação serão apresentados a seguir.

Considerando a individualidade de cada parceiro, o bem-estar subjetivo (BES) é uma noção contemporânea que tem sido concebida na literatura como o estudo científico da felicidade (Nunes, Hutz, \& Giacomoni, 2009; Reppold, Giacomoni, \& Hutz, 2007; Scorsolini-Comin $\&$ Santos, 2010; Seligman, 2002). Trata-se de uma experiência interna de cada indivíduo, que emite um julgamento de como ele se sente e o seu grau de satisfação com a vida. De acordo com a literatura, pessoas casadas de ambos os sexos relatam mais felicidade do que aquelas que nunca casaram ou são divorciadas, separadas ou viúvas. Pessoas que coabitam com um parceiro também são significativamente mais felizes em algumas culturas do que aquelas que vivem sozinhas (Diener \& Lucas, 2000; Lee, Seccombe, \& Shehan, 1991; Seligman, 2002). A relação entre bem-estar subjetivo e ser casado se aplica a pessoas de todas as idades, níveis de renda e graus de instrução, bem como origens étnico-raciais, sendo a qualidade conjugal associada positivamente ao bem-estar pessoal (Snyder \& Lopez, 2009).

Por satisfação conjugal (SC), entende-se a atitude em relação a aspectos do cônjuge e da interação conjugal (Dela Coleta, 1989). Para Wagner e Falcke (2001), no conceito de SC estão implicadas tanto as experiências precoces do sujeito na sua família, como os aspectos vivenciais da relação diádica atual, além das variáveis de personalidade e biodemográficas. Na tentativa de explicação do fenômeno amoroso e conjugal, uma diversidade de posições teóricas tem sido proposta, o que se justifica pela multiplicidade de variáveis implicadas. Isso ocorre, também, devido ao fato de o casamento ser um momento em que a porta da família se abre para a introdução de um novo membro, oriundo de um outro sistema familiar (Mosmann et al., 2006).

No Brasil, os estudos sobre o BES começaram a ter maior destaque na primeira década de 2000 (Paludo \& Koller, 2007; Scorsolini-Comin, 2009; Scorsolini-Comin \& Santos, 2009), impulsionados pela ascensão da Psicologia Positiva nos EUA e na Europa. Esta corrente se dedica ao enfoque científico e aplicado das qualidades das pessoas e da promoção de seu funcionamento positivo (Park \& Peterson, 2007; Snyder \& Lopez, 2009). Ainda segundo esses autores, a ciência e a prática da Psicologia Positiva estão direcionadas para a identificação e a compreensão das qualidades e virtudes humanas, bem como para auxiliar no sentido de que as pessoas tenham vidas mais felizes e produtivas (Calvetti, Muller, \& Nunes, 2007).
A literatura estabelece um claro consenso acerca dos fatores que compõem o BES: satisfação com a vida e afetos positivos e negativos (Diener, 1996). Afeto positivo corresponde a um estado de contentamento puro experimentado em um determinado momento como um estado de alerta, de entusiasmo e de atividade. Afeto negativo refere-se a um estado de distração e engajamento desprazível que também é transitório, mas que inclui emoções desagradáveis. A satisfação com a vida é um julgamento cognitivo de algum domínio específico na vida da pessoa, um processo de juízo e avaliação geral da própria vida. Esses três fatores constituem os fundamentos para a construção de alguns dos instrumentos de mensuração do BES, como a Escala de Bem-estar Subjetivo - EBES (Albuquerque \& Tróccoli, 2004), que será objeto do presente estudo.

Como a literatura aponta que pessoas casadas são mais felizes (Seligman, 2002; Snyder \& Lopez, 2009), hipotetiza-se que existam correlações positivas e significativas entre o BES e a SC. No entanto, não foram encontradas na literatura evidências científicas obtidas a partir da utilização de escalas de avaliação do bem-estar individual e da SC. A despeito do caráter complementar que reveste essas facetas do indivíduo, os estudos disponíveis avaliaram tais dimensões de forma separada ou por meio de outras noções, como empatia, habilidades sociais (Sardinha, Falcone, \& Ferreira, 2009), resiliência (Dell'Aglio, Koller, \&Yunes, 2006) ou mesmo a partir de revisão da literatura (Albuquerque \& Tróccoli, 2004; Passareli \& Silva, 2007). Para o avanço do conhecimento nessa área, é imperioso que se avalie de que modo essas dimensões se articulam e se influenciam mutuamente, como traremos a seguir.

Isso posto, foram construídas as seguintes hipóteses: (a) A satisfação com a vida (fator do BES) está positiva e significativamente correlacionada com a satisfação com os aspectos emocionais do cônjuge (fator da SC); (b) A satisfação com a vida (fator do BES) está positiva e significativamente correlacionada com a satisfação acerca dos aspectos estruturais do cônjuge (fator da SC); (c) Os afetos positivos (fator do BES) estão positivamente correlacionados com a interação conjugal (fator da SC).

\section{Método}

\section{Participantes}

Em relação aos critérios de inclusão/exclusão, foram definidos para inclusão de participantes no estudo: estar consensualmente casado há, no mínimo, um ano; não apresentar indícios de comprometimento cognitivo ou comportamental; não estar em processo de separação conjugal. Não se restringiu a duração máxima para os casamentos, idade, ocupação, classificação socioeconômica ou quantidade de filhos.

Os participantes foram selecionados pela técnica da "bola de neve", em que novos voluntários (tanto cônju- 
ges, como outros casais) foram indicados pelos próprios respondentes, a partir dos contatos do pesquisador. O primeiro casal participante foi localizado na própria universidade em que se realizou a pesquisa, por meio da rede de contatos do pesquisador, que lhes apresentou o estudo e os seus objetivos. Em cada uma das aplicações, após serem esclarecidos sobre a pesquisa, os participantes assinaram o Termo de Consentimento Livre e Esclarecido e preencheram os questionários e escalas. A aplicação da bateria de instrumentos foi individual, ou seja, mesmo se tratando de casais, cada membro respondeu de modo independente (sem a presença do cônjuge). Outro cuidado tomado para assegurar a aplicação independente dos instrumentos foi de que os respondentes não tivessem acesso às respostas do parceiro (contato prévio com os instrumentos respondidos, ou conversa sobre os mesmos após a aplicação), o que poderia influenciar nas respostas. Assim, a aplicação com cada cônjuge deu-se de modo sequenciado e independente.

Neste estudo, não foram estudadas as correlações intradíades (grau de consenso entre os membros do par), mas sim as respostas dadas de modo independente por pessoas casadas.

\section{Amostragem}

A saturação da amostra foi definida por meio dos procedimentos preconizados por Krejcie e Morgan (1970). De acordo com esses autores, pode-se estimar o $n$ da amostra a partir do $N$ do universo (no caso, pessoas casadas da Universidade), para uma probabilidade de erro nunca superior a 5\%. Assim, podemos afirmar que o $n$ da amostra aumenta à medida que o $N$ da população é mais elevado, sendo, no entanto, esse aumento representado por uma curva algorítmica e não linear.

Sendo assim, a amostra foi do tipo não probabilística, composta por critérios de conveniência, totalizando 106 participantes (membros de 53 casais heterossexuais), legalmente casados há, no mínimo, um ano, com ou sem filhos. No que concerne ao perfil sociodemográfico dos participantes, a média de idade foi de $42 \pm 11$ anos. A média de idade dos homens foi de 43,4 anos, ao passo que a das mulheres foi de 40,7. No que se refere ao tempo de união, a média em anos de casamento foi de 16,11 $\pm 11,35$. Em relação ao número de filhos dos casais participantes, a média foi de $1,49( \pm 1,22)$ filhos para cada participante. Em termos da classificação socioeconômica, definida a partir do critério da Associação Brasileira dos Institutos de Pesquisa de Mercado (Abipeme, 1997), a maioria dos participantes pertencia à classe $\mathrm{B}(60,37 \%)$. Acompanhando a classificação do status socioeconômico, a renda per capita foi de 5,03( $\pm 3,63)$ salários. Em relação ao grau de instrução dos participantes, a maioria $(66,98 \%)$ possuía nível superior, o que se observou tanto entre homens quanto entre mulheres.

\section{Instrumentos}

Questionário Sociodemográfico. Desenvolvido pelos autores deste estudo para identificação do participante e de suas características demográficas e socioeconômicas. Contém questões como renda, trabalho, idade, grau de instrução, número de filhos e tempo de relacionamento.

Escala Abipeme de Classificação Socioeconômica. O critério Abipeme (1997) é uma escala ou classificação socioeconômica aferida por intermédio da atribuição de pesos a um conjunto de itens de conforto doméstico, além do nível de escolaridade do chefe de família. A classificação socioeconômica da população é apresentada por meio de cinco classes, denominadas A, B, C, D e E, que correspondem, respectivamente, a uma pontuação determinada pelo instrumento.

Escala de Bem-estar Subjetivo (EBES; Albuquerque \& Tróccoli, 2004). Trata-se de um instrumento inspirado em escalas existentes no exterior: Escala de Afeto Positivo e Afeto Negativo (PANAS; Watson, Clark, \& Tellegen, 1988), Escala de Satisfação com a Vida (SWLS; Diener, Emmons, Larsen, \& Griffin, 1985) e Escala de Bem-estar Subjetivo (SWBS; Lawrence, \& Liang, 1988), com itens elaborados e analisados em grupos de validação semântica. A análise dos componentes principais e a análise fatorial (extração dos eixos principais - $P A F$ e rotação oblimin) revelaram três fatores: afeto positivo (21 itens, explicando $24,3 \%$ da variância, alfa de Cronbach $=0,95$ ); afeto negativo (26 itens, 24,9\% da variância, alfa de Cronbach $=0,95)$ e satisfação-insatisfação com a vida (15 itens, 21,9\% da variância, alfa de Cronbach =0,90). Juntos, os três fatores explicaram $44,1 \%$ da variância total do construto (Albuquerque \& Tróccoli, 2004).

Os itens de 1 a 47 descrevem os fatores afetos positivos e negativos, devendo o sujeito responder como tem se sentido ultimamente em uma escala Likert de cinco pontos, na qual 1 significa "nem um pouco" e 5 significa "extremamente". Os itens 48 a 62 descrevem julgamentos relativos à avaliação de satisfação ou insatisfação com a vida, com alternativas de resposta de cinco pontos, na qual 1 significa "discordo plenamente" e 5 significa "concordo plenamente".

Escala de Satisfação Conjugal (ESC). É um instrumento de origem mexicana (Pick de Weiss \& Andrade Palos, 1988), adaptado por Dela Coleta (1989) para o contexto brasileiro no final da década de 1980, que compreende a satisfação conjugal como a atitude em relação a aspectos do cônjuge e da interação conjugal. Trata-se de uma escala composta por três fatores acerca de aspectos da satisfação conjugal: satisfação com a interação conjugal (10 itens referentes à união entre os parceiros, compartilhamento de emoções e tarefas, com alfa de Cronbach de 0,86); satisfação com os aspectos emocionais do cônjuge (10 itens que mensuram a percepção acerca das emoções do parceiro, com alfa de Cronbach de 0,81 ); e satisfação com os aspectos estruturais do cônjuge ( 9 itens que avaliam a forma de organização e de 
estabelecimento e cumprimento de regras pelo cônjuge, com alfa de Cronbach de 0,79) (Dela Coleta, 1989).

\section{Análise dos Dados}

Os dados obtidos com a aplicação dos instrumentos foram transpostos para o Software SAS 9.2 e categorizados a partir de números de identificação, por casal, por sexo e, consequentemente, por participante. Foram calculadas as correlações entre os fatores: (a) afetos positivos; (b) afetos negativos; (c) satisfação com a vida (a, b, c são fatores do BES); (d) interação conjugal; (e) aspectos emocionais; (f) aspectos estruturais (d, e, f são fatores de satisfação conjugal, segundo a ESC). Como o objetivo da análise foi comparar parâmetros, optou-se por métodos paramétricos. Esses métodos implicam em construir um modelo a partir dos dados observados, esperando-se que o resíduo, ou erro, tenha distribuição normal, e não os dados, pois os mesmos são explicados a partir de diversas variáveis. A força da grandeza do coeficiente de correlação foi avaliada conforme procedimento proposto por Zou, Tuncali e Silverman (2003). O nível de significância adotado foi de $p<0,05$.

Depois da definição das correlações, estabeleceu-se a relação dos fatores a partir de um modelo linear múltiplo em que todos os fatores foram considerados a princípio, ou seja, cada fator (do BES e da SC) era considerado uma variável dependente (VD), ao passo que todos os demais eram assumidos como variável independente (VI). Assim, todos os fatores foram correlacionados entre si, tanto como VD como VI, uma vez que não se podia afirmar, pela literatura, se havia uma direção unívoca dos fatores. Portanto, primeiramente fizemos uma análise univariada para entender as rela- ções separadamente e, depois, procuramos observar as mesmas sob o ponto de vista multivariado, ou seja, não mais separadamente, mas sim na presença dos demais fatores. Com a adoção desse procedimento, pudemos compreender a direção das correlações e verificarmos aquelas mais diretamente relacionadas a cada um dos fatores. Desse modo, para estabelecer o modelo final, todos os fatores (a, b, c, d, e, f) foram submetidos ao método de seleção de variáveis stepwise, no qual permaneceram no modelo aqueles que apresentaram maior evidência de significância do ponto de vista multivariado. O uso deste método permitiu "filtrar", dentre todos os fatores elencados, os que tinham maior poder de predição, uma vez que se trata de uma ferramenta de análise adequada para examinar e entender todos os tipos de relacionamentos interdependentes (Hair, Anderson, Tatham, \& Black, 1995; Montgomery, Vining, \& Peck, 2001; Siegel \& Castellan, 1988).

\section{Cuidados Éticos}

Este estudo foi aprovado pelo Comitê de Ética em Pesquisa da Faculdade de Filosofia, Ciências e Letras de Ribeirão Preto, Universidade de São Paulo (processo ${ }^{0}$ 349/2007 - 2007.1.2016.59.0) e obedeceu rigorosamente aos princípios que regem a pesquisa envolvendo seres humanos.

\section{Resultados}

A partir dos resultados obtidos, serão examinadas as correlações entre os fatores dos construtos. A Tabela 1 resume as correlações e $p$-values encontrados pela associação dos fatores elencados no presente estudo.

Tabela 1

Matriz de Correlação e p-values Independentemente de Sexo $(N=106)$

\begin{tabular}{lcccccc}
\hline & $\begin{array}{c}\text { Afetos } \\
\text { positivos }\end{array}$ & $\begin{array}{c}\text { Afetos } \\
\text { negativos }\end{array}$ & $\begin{array}{c}\text { Satisfação } \\
\text { com a vida }\end{array}$ & $\begin{array}{c}\text { Interação } \\
\text { conjugal }\end{array}$ & $\begin{array}{c}\text { Aspectos } \\
\text { emocionais }\end{array}$ & $\begin{array}{c}\text { Aspectos } \\
\text { estruturais }\end{array}$ \\
\hline Afetos positivos & - & $-0,45(<0,01)$ & $0,19(0,05)$ & $-0,30(<0,01)$ & $-0,18(0,06)$ & $-0,15(0,14)$ \\
Afetos negativos & & - & $0,09(0,35)$ & $0,31(<0,01)$ & $0,23(0,02)$ & $0,20(0,04)$ \\
Satisfação com a vida & & & - & $0,03(0,77)$ & $0,06(0,54)$ & $0,06(0,53)$ \\
Interação conjugal & & & & - & $0,53(<0,01)$ & $0,62(<0,01)$ \\
Aspectos emocionais & & & & & - & $0,56(<0,01)$ \\
Aspectos estruturais & & & & & & - \\
\hline
\end{tabular}

Para organizar didaticamente a apresentação dos resultados, primeiramente, serão consideradas as correlações entre os fatores do BES. Em relação aos afetos positivos e negativos, independentemente do sexo, encontrou-se uma correlação negativa $(r=-0,45)$, considerada moderada (Zou et al., 2003). No estudo de Albuquerque e Tróccoli (2004), foi encontrado o coeficiente de $-0,36$, o que se situa na mesma faixa de correlação. Esse resultado de correlação negativa pode ser compreendido tendo-se em mente que, quanto mais uma pessoa apresenta afetos positivos, menores devem ser os seus afetos negativos. O $p$-value encontrado nessa relação foi de $p<0,01$, o que é considerado significativo. Desse modo, podemos afirmar que tais fatores são negativamente correlacionados, 
o que é corroborado pela análise de regressão múltipla e pela literatura da área (Albuquerque \& Tróccoli, 2004; Lawrence \& Liang, 1988; Seligman, 2002).

Correlacionando-se os afetos positivos com o terceiro fator do BES, ou seja, a satisfação com a vida, encontramos a correlação $r=0,19$, considerada leve, com nível de significância $p=0,05$. No estudo de Albuquerque e Tróccoli (2004), essa correlação foi de $r=0,53$, o que é considerada moderada. Assim, no presente estudo, esses fatores apresentaram menor correlação do que a obtida no estudo de validação da escala. A análise de regressão múltipla da amostra revelou que os afetos positivos estão relacionados, de maneira forte e significativa, à satisfação com a vida. Desse modo, no presente estudo foram encontradas correlações significativas entre os três fatores do BES, o que permite afirmar que a EBES mostrouse adequada e válida também para a amostra de pessoas casadas. No estudo original (Albuquerque \& Tróccoli, 2004) não foram avaliadas apenas pessoas casadas, mas também solteiras.

Acerca das correlações estabelecidas entre os fatores da satisfação conjugal, verificou-se que os aspectos emocionais foram positivamente correlacionados com os aspectos estruturais $(r=0,56 ; p<0,01)$, ou seja, aspectos emocionais e estruturais apresentaram correlação de moderada a forte. Essa correlação foi confirmada pela análise de regressão múltipla. Tal constatação corrobora os estudos de Dela Coleta (1989) e Pick de Weiss e Andrade Palos (1988), que validaram o instrumento de investigação da satisfação conjugal utilizado nos contextos brasileiro e mexicano, respectivamente.

Ainda de acordo com os dados obtidos também se verificou correlação positiva e significativa entre os aspectos emocionais e a interação conjugal ( $r=0,53$; $p<0,01)$, fatores da SC. Em relação aos aspectos estruturais e a interação conjugal, também fatores da SC, a correlação encontrada foi de moderada a forte $(r=0,62$; $p<0,01$ ), o que indica grande coesão entre os fatores, o que foi destacado também pela regressão múltipla. Assim, pode-se afirmar que a correlação entre aspectos estruturais e interação conjugal foi significativa.

Correlacionando-se os fatores das duas escalas de avaliação, pode-se observar que os afetos positivos (fator do BES) estão negativamente correlacionados $(r=-0,18)$ com os aspectos emocionais dos cônjuges (fator da $\mathrm{SC}$ ), com nível de significância $p=0,06$. Essa correlação, pela classificação de Zou et al. (2003), é considerada entre fraca e sem associação. Correlacionando os afetos positivos com o segundo fator da SC, ou seja, os aspectos estruturais do cônjuge, também encontramos uma correlação negativa $(r=-0,15)$ e não significativa $(p=0,14)$.

Em relação ao último fator da SC, ou seja, a interação conjugal, a correlação com os afetos positivos (fator do BES) foi de $r=-0,30$, com nível de significância de $p<0,01$. Assim, os afetos positivos, em relação à $\mathrm{SC}$, só se mostraram correlacionados com a interação conjugal (correlação negativa), não apresentando associações com os aspectos emocionais e estruturais. Tal achado não confirmou a terceira hipótese do estudo (em dois sentidos, tanto assumindo o BES como VD e a interação conjugal como VI, e vice-versa), uma vez que a correlação estabelecida foi negativa, ou seja, quanto maior o nível de afetos positivos, menor a satisfação com a interação conjugal.

A Tabela 2 resume os resultados obtidos com o emprego da análise de regressão múltipla pelo método de seleção de variáveis stepwise.

Tabela 2

Resultados do Modelo de Regressão Ajustado utilizando o Método de Seleção de Variáveis Stepwise, com Estimativa do Parâmetro definida por meio do Método de Mínimos Quadrados (Least Mean Square Method) (N=106)

\begin{tabular}{llcc}
\hline $\begin{array}{l}\text { Variável } \\
\text { Resposta }\end{array}$ & $\begin{array}{l}\text { Variáveis } \\
\text { Explicativas }\end{array}$ & $\begin{array}{c}\text { Estimativa } \\
\text { do parâmetro }\end{array}$ & $p$-valor \\
\hline Afetos positivos & Afetos negativos & $-0,35$ & 0,01 \\
& Satisfação com a vida & 0,91 & 0,01 \\
& Aspectos estruturais & 0,56 & 0,08 \\
Afetos negativos & Afetos positivos & $-0,55$ & 0,01 \\
& Satisfação com a vida & 0,94 & 0,01 \\
Satisfação com a vida & Afetos positivos & 0,11 & 0,01 \\
Interação conjugal & Afetos negativos & 0,06 & 0,01 \\
Aspectos emocionais & Aspectos estruturais & 0,59 & 0,01 \\
Aspectos estruturais & Aspectos estruturais & 0,34 & 0,01 \\
& Interação conjugal & 0,34 & 0,01 \\
& Aspectos emocionais & 0,35 & 0,01 \\
\hline
\end{tabular}

De acordo com os resultados da análise de regressão múltipla, os afetos positivos não se relacionaram significativamente com quaisquer fatores da $\mathrm{SC}$, ou seja, ne- nhum dos fatores da SC contribuiu para explicar os afetos positivos. De modo similar, os afetos positivos não contribuíram para explicar quaisquer dos fatores da SC. 
Tais achados rejeitam a terceira hipótese do estudo. Há que se problematizar que os fatores da SC se referem, fundamentalmente, a uma avaliação que é feita acerca do outro cônjuge, ou seja, a satisfação vai se dar em função da percepção que se tem do mesmo, e não de aspectos do próprio avaliador.

Os aspectos emocionais (fator da SC) apresentaram correlação de $r=0,23 \mathrm{com}$ os afetos negativos (fator do $\mathrm{BES}$ ), com $p$-value 0,02 , o que é considerado significativo. Tal correlação moderada não foi encontrada quando se relacionaram afetos positivos (fator do BES) e aspectos emocionais (fator da $\mathrm{SC}$ ). Em termos de aspectos estruturais (fator da SC), também se registrou uma correlação positiva de 0,20 , considerada significativa $(p=0,04)$ e leve (Zou et al., 2003). No que diz respeito à interação conjugal (fator da SC), a correlação com os afetos negativos foi de 0,31 (moderada), com nível de significância $p<0,01$.

Desse modo, os afetos negativos estão positivamente correlacionados com todos os fatores da SC (aspectos emocionais, aspectos estruturais e interação conjugal), embora a análise de regressão múltipla revele que nenhum desses fatores interfere diretamente nos afetos negativos, ou seja, não se tratam de correlações significativas ou que expliquem os afetos negativos. Similarmente, os afetos negativos não interferem significativamente em quaisquer dos fatores da SC, pela análise multivariada.

A satisfação com a vida (fator do BES) não apresentou correlação significativa com quaisquer dos fatores da SC: satisfação com os aspectos emocionais $(r=0,06 ; p=0,54)$; satisfação com os aspectos estruturais $(r=0,06 ; p=0,53)$; satisfação com a interação conjugal $(r=0,03 ; p=0,77)$, rejeitando as duas primeiras hipóteses do estudo. Ou seja, tanto pela análise univariada quanto pela multivariada não foi possível estabelecer associações entre os fatores satisfação com a vida (fator do BES) e os aspectos emocionais do cônjuge (fator da SC) (primeira hipótese), nem da satisfação com a vida (fator do BES) com os aspectos estruturais do cônjuge (fator da SC) (segunda hipótese), tanto assumindo a satisfação com a vida como VD (e os fatores da SC como VI) como os fatores da SC como VD e a satisfação com a vida como VI.

$\mathrm{O}$ BES e a SC (fatores gerais) também não apresentaram associações significativas. Todavia, em relação aos fatores que compõem esses construtos, como mencionado anteriormente, pode-se destacar que os afetos positivos estão correlacionados com a interação conjugal (correlação negativa, de fraca a moderada) e os afetos negativos com os aspectos emocionais, estruturais e a interação conjugal (correlações de fracas a moderadas).

\section{Discussão}

Segundo Seligman (2002), a relação entre emoção positiva e emoção negativa não é de total oposição. Essa é uma das questões centrais da Psicologia Positiva e que ainda não foi suficientemente investigada. Como somos constituídos tanto por afetos positivos como negativos, Diener (1996) destaca que eles não seriam opostos nem excludentes. No entanto, para uma pessoa ser feliz, é fundamental que os afetos positivos se sobreponham aos negativos.

Pelos dados do presente estudo, pode-se afirmar que afetos positivos e negativos são negativa e significativamente correlacionados, corroborando a afirmação de Diener (1996) e de outros pesquisadores contemporâneos do BES, inclusive no Brasil (Albuquerque \& Tróccoli, 2004), o que não significa que um anule a existência do outro, uma vez que ninguém apresenta nível nulo de afetos, quer sejam positivos ou negativos.

Nesse sentido, a terceira hipótese (correlação entre afetos positivos do BES e satisfação com a interação conjugal da SC) não foi confirmada. Não encontramos aporte na literatura para fundamentar tal consideração.

Pelos dados do estudo, pode-se pensar que os afetos negativos estariam relacionados ao modo como as pessoas estabelecem seus relacionamentos conjugais, mas que a satisfação conjugal não dependeria do nível de afetos negativos de um cônjuge ou da díade, mas do nível de afetos positivos. A avaliação da satisfação com a vida (terceiro fator do BES) é subjetiva e, como tal, reflete as expressões de cada pessoa quanto aos seus próprios critérios de satisfação com a vida como um todo e em domínios específicos, como saúde, trabalho, condições de moradia, relações sociais e outros. Assim, reflete o bem-estar individual, o modo e os motivos que levam as pessoas a viverem suas experiências de vida de maneira positiva.

O fato de a satisfação com a vida (fator do BES) não estar correlacionada com quaisquer fatores da $\mathrm{SC}$ atesta que uma percepção positiva acerca da vida não está, necessariamente, associada a uma percepção igualmente positiva acerca do cônjuge, o que sugere a necessidade de considerarmos que a atitude em relação ao companheiro nem sempre é modificada se o indivíduo apresenta uma atitude positiva ou de prazer diante da vida.

A satisfação com a vida não se mostrou relacionada com quaisquer dos fatores da SC (do mesmo modo, nenhum dos fatores da SC se correlacionou com este fator do BES). Assim, não se pode afirmar, de modo inequívoco, que os construtos BES e SC não estejam correlacionados, embora fatores específicos de cada um dos construtos estejam correlacionados entre si e de maneira significativa, o que nem sempre é reportado na literatura científica, que privilegia as correlações em termos dos construtos gerais (fatores gerais).

\section{Conclusões}

Retomando as hipóteses do presente estudo, todos os fatores do BES mostraram-se correlacionados entre si, o que foi observado também em relação aos fatores da SC. Esses achados permitem considerar que os construtos avaliados, bem como os instrumentos utilizados para 
mensurá-los, apresentaram boa consistência de medidas nesta investigação.

Considerando o escopo deste estudo, podemos apontar que a satisfação com a vida (fator do BES) não está correlacionada significativamente com a satisfação acerca dos aspectos emocionais e estruturais do cônjuge (fatores da $\mathrm{SC}$ ), nem que tais fatores se correlacionem à satisfação com a vida. Assim, podemos afirmar que a satisfação em termos individuais (mensurada por meio do fator satisfação com a vida, do BES) não se associa à satisfação com o parceiro (mensurada por meios dos fatores da SC). Futuramente, para aprofundar tal achado, a avaliação de cada um dos casais (díades) acerca desses mesmos fatores poderá revelar se existiria mesmo um BES do casal, ou seja, na perspectiva de cada casal quais seriam as correlações existentes entre os fatores do BES.

Do ponto de vista teórico-conceitual, podemos examinar os resultados obtidos a partir dos pressupostos da Psicologia Positiva (Scorsolini-Comin \& Santos, 2010). Primeiramente, a busca pelos aspectos adaptativos do ser humano está presente desde a construção dos fatores dos instrumentos, uma vez que eles destacam a satisfação e os aspectos que fazem com que as pessoas estejam juntas e permaneçam vinculadas a partir de um relacionamento afetivo. Em termos do BES, a Psicologia Positiva se debruça, notadamente, nos afetos positivos e no modo como eles repercutem na avaliação subjetiva de bem-estar e de satisfação. Pelos dados deste estudo, pode-se sugerir que tal referencial se detém nos aspectos fundamentalmente adaptativos da noção de satisfação conjugal, embora pudesse considerar também o movimento oposto a essa coesão.

Como já destacado por Diener (1996), o nível de satisfação pode ser um modulador das emoções, aumentando ou diminuindo as emoções positivas ou negativas dependendo do que o indivíduo pensa, o que pode ser estendido à instituição casamento. $\mathrm{O}$ modo como cada cônjuge participa da constituição do estar junto em uma relação, compartilhando projetos, sentimentos, desapontamentos, diferenças e intimidade, pode ser um fator de satisfação não apenas com o domínio conjugal, como também com a própria vida. A correlação encontrada entre os fatores de interação conjugal e os aspectos estruturais do casamento (fatores da SC) sugere que tais sentimentos e percepções estariam no cerne da estrutura do relacionamento conjugal, embora não se relacionem com a satisfação com a vida (fator do BES), ou seja, com a percepção que o indivíduo tem sobre a sua própria vida. Assim, sugerimos a possibilidade de que as medidas de BES considerem também os aspectos relacionais e intersubjetivos, e não apenas os centrados no indivíduo.

$\mathrm{O}$ que se pretende com a perspectiva da Psicologia Positiva é justamente oferecer um horizonte para que as características positivas do psiquismo (do indivíduo e seu companheiro) possam ser reconhecidas e lapidadas tanto pelos casais que atribuem determinadas qualidades aos seus relacionamentos conjugais, quanto pelos pesquisa- dores, que devem estar abertos às contradições, a fim de contribuir para que essas características adaptativas aflorem de modo saudável, flexível e como promotoras de bem-estar e felicidade.

\section{Referências}

Albuquerque, A. S., \& Tróccoli, B. T. (2004). Desenvolvimento de uma escala de bem-estar subjetivo. Psicologia: Teoria e Pesquisa, 20(2), 153-164.

Associação Brasileira dos Institutos de Pesquisa de Mercado. (1997). Critério de classificação socioeconômica - Brasil (CCSEB). São Paulo, SP: Autor.

Calvetti, P. U., Muller, M. C., \& Nunes, M. L. T. (2007). Psicologia da Saúde e Psicologia Positiva: Perspectivas e desafios. Psicologia: Ciência e Profissão, 27(4), 706-717.

Dela Coleta, M. F. (1989). A medida da satisfação conjugal: Adaptação de uma escala. Psico, 18(2), 90-112.

Dell'Aglio, D. D., Koller, S. H., \& Yunes, M. A. (2006). Resiliência e Psicologia Positiva: Interfaces do risco à proteção. São Paulo, SP: Casa do Psicólogo.

Diener, E. (1996). Traits can be powerful, but are not enough: Lessons from Subjective Well-Being. Journal of Research in Personality, 30(1), 389-399.

Diener, E., Emmons, R. A., Larsen, R. J., \& Griffin, S. (1985). The satisfaction with life scale. Journal of Personality Assessment, 49, 71-75.

Diener, E., \& Lucas, R. E. (2000). Explaining differences in societal levels of happiness: Relative standards, need fulfillment, culture and evaluation theory. Journal of Happiness Studies, 1, 41-78.

Féres-Carneiro, T. (1998). Casamento contemporâneo: O difícil convívio da individualidade com a conjugalidade. Psicologia: Reflexão e Crítica, 11(2), 379-394.

Hair, J. F. J., Anderson, R. E., Tatham, R. L., \& Black, W. C. (1995). Multivariate data analysis with readings $\left(4^{\text {th }}\right.$ ed.). Saddle River, NJ: Prentice Hall.

Jablonski, B. (2001). Atitudes frente ao fim do casamento. In T. Féres-Carneiro (Ed.), Casamento e família: Do social à clínica (pp. 81-95). Rio de Janeiro, RJ: Nau.

Krejcie, R. V., \& Morgan, D. W. (1970). Determining sample size for research activities. Educational and Psychological Measurement, 30, 607-610.

Lawrence, R. H., \& Liang, J. (1988). Structural integration of the Affect Balance Scale and the Life Satisfaction Index A: Race, sex, and age differences. Psychology and Aging, 3, 375-384.

Lee, G. R., Seccombe, K., \& Shehan, C. L. (1991). Marital status and personal happiness: An analysis of trend data. Journal of Marriage and the Family, 53, 839-844.

Mosmann, C., Wagner, A., \& Féres-Carneiro, T. (2006). Qualidade conjugal: Mapeando conceitos. Paideia, 16(35), 315325.

Montgomery, D., Vining, G., \& Peck, E. A. (2001). Introduction to linear regression analysis $\left(3^{\text {nd }}\right.$ ed.). New York, NY: John Wiley Profession.

Nunes, C. H. S., Hutz, C. S., \& Giacomoni, C. H. (2009). Associação entre bem-estar subjetivo e personalidade no modelo dos cinco grandes fatores. Avaliação Psicológica, 8(1), 99-108.

Paludo, S. S., \& Koller, S. H. (2007). Psicologia Positiva: Uma nova abordagem para antigas questões. Paideia, 17(36), 9-20. 
Park, N., \& Peterson, C. (2007). Methodological issues in Positive Psychology and the assessment of character strengths. In A. D. Ong \& M. H. M. Van Dulmen (Eds.), Oxford handbook of methods in Positive Psychology (pp. 292305). New York, NY: Oxford University Press.

Passareli, P. M., \& Silva, J. A. (2007). Psicologia Positiva e o estudo do bem-estar subjetivo. Estudos de Psicologia (Campinas), 24(4), 513-517.

Pick de Weiss, S., \& Andrade Palos, P. (1988). Desarrolo y validacion de la escala de satisfaccion marital. Psiquiatria, 1, 9-20.

Reppold, C. T., Giacomoni, C. H., \& Hutz, C. S. (2007). Bemestar subjetivo: Definição e formas de avaliação. In P. Schelini (Ed.), Alguns domínios da avaliação psicológica (pp. 111124). Campinas, SP: Alínea.

Sardinha, A., Falcone, E. M. O, \& Ferreira, M. C. (2009). As relações entre a satisfação conjugal e as habilidades sociais percebidas no cônjuge. Psicologia: Teoria e Pesquisa, 25(3), 329-336.

Scorsolini-Comin, F. (2009). Casar, verbo (in)transitivo: Conjugalidade, bem-estar subjetivo e satisfação conjugal na perspectiva da Psicologia Positiva. Dissertação de Mestrado não-publicada, Faculdade de Filosofia, Ciências e Letras, Universidade de São Paulo, Ribeirão Preto, SP.

Scorsolini-Comin, F., \& Santos, M. A. (2009). A Psicologia Positiva no contexto brasileiro: História, presente e perspectivas futuras. In S. N. Jesus, I. Leal, \& M. Rezende (Eds.), Experiências e intervenções em Psicologia da Saúde (pp. 862878). Faro, Portugal: Universidade do Algarve.

Scorsolini-Comin, F., \& Santos, M. A. (2010). The scientific study of happiness and health promotion: An integrative literature review. Revista Latino-Americana de Enfermagem, $18(3), 472-479$.

Seligman, M. E. (2002). Authentic happiness: Using the new Positive Psychology to realize your potential for lasting fulfilment. London: Nicholas Brealey.

Siegel, S., \& Castellan, N. J. (1988). Nonparametric statistics for the behavioral sciences $\left(2^{\text {nd }}\right.$ ed.). New York, NY: McGraw-Hill College.

Snyder, C. R., \& Lopez, S. J. (2009). Psicologia Positiva: Uma abordagem científica e prática das qualidades humanas $(\mathrm{R}$. C. Costa, Trad.). São Paulo, SP: Artmed.

Wagner, A., \& Falcke, D. (2001). Satisfação conjugal e transgeracionalidade: Uma revisão teórica sobre o tema. Psicologia Clínica, 13(2), 1-15.

Watson, D., Clark, L. A., \& Tellegen, A. (1988). Development and validation of brief measures of positive and negative affect: The PANAS scales. Journal of Personality and Social Psychology, 54, 1063-1067.

Zou, K. H., Tuncali, K., \& Silverman, S. G. (2003). Correlation and simple linear regression. Radiology, 227, 617-628. 